\title{
Conserved Modules Required for Drosophila TRP Function in Vivo
}

\author{
${ }^{(1)}$ Zijing Chen, ${ }^{1,2}$ Maggie Kerwin, ${ }^{1,2}$ Orlaith Keenan, ${ }^{1,2}$ and ${ }^{\circledR}$ Craig Montell ${ }^{1,2}$ \\ ${ }^{1}$ Department of Molecular, Cellular, and Developmental Biology, University of California, Santa Barbara, Santa Barbara, California 93106, and \\ ${ }^{2}$ Neuroscience Research Institute, University of California, Santa Barbara, Santa Barbara, California 93106
}

Transient receptor potential (TRP) channels are broadly required in animals for sensory physiology. To provide insights into regulatory mechanisms, the structures of many TRPs have been solved. This has led to new models, some of which have been tested in vitro. Here, using the classical TRP required for Drosophila visual transduction, we uncovered structural requirements for channel function in photoreceptor cells. Using a combination of molecular genetics, field recordings, protein expression analysis, and molecular modeling, we interrogated roles for the S4-S5 linker and the TRP domain, and revealed mutations in the S4-S5 linker that impair channel opening or closing. We also uncovered differential requirements for the two highly conserved motifs in the TRP domain for activation and protein stability. By performing genetic complementation, we found an intrasubunit interaction between the S4-S5 linker and the S5 segment that contributes to activation. This analysis highlights key structural requirements for TRP channel opening, closing, folding, and for intrasubunit interactions in a native context-Drosophila photoreceptor cells.

Key words: TRP channels; TRPC; Drosophila; phototransduction; vision; electroretinogram

\section{Significance Statement}

The importance of TRP channels for sensory biology and human health has motivated tremendous effort in trying to understand the roles of the structural motifs essential for their activation, inactivation, and protein folding. In the current work, we have exploited the unique advantages of the Drosophila visual system to reveal mechanistic insights into TRP channel function in a native system-photoreceptor cells. Using a combination of electrophysiology (field recordings), cell biology, and molecular modeling, we have revealed roles of key motifs for activation, inactivation and protein folding of TRP in vivo.

\section{Introduction}

Transient receptor potential (TRP) channels comprise a large family of cation channels that have critical roles in the senses ranging from vision to taste, hearing, temperature sensation, touch, and proprioception (Venkatachalam and Montell, 2007). Multiple diseases result from mutations in TRPs, including pain insensitivity, severe itching, and neurodegeneration (Gees et al., 2012; Moore et al., 2018).

The canonical TRP subfamily, TRPCs, is defined by Drosophila TRP, which functions in photoreceptor cells (Montell and Rubin, 1989; Hardie and Minke, 1992). The first mammalian TRP homologs identified, TRPC1 and TRPC3, fall into the TRPC subfamily

\footnotetext{
Received Jan. 27, 2021; revised May 21, 2021; accepted May 25, 2021.

Author contributions: C.M. and Z.C. designed research; Z.C., M.K. and 0.K. performed research; C.M. and Z.C. analyzed data; C.M. and Z.C. wrote the paper.

This work was supported by a grant to C.M. from the National Eye Institute (EY010852). We thank the Drosophila Genomics Resource Center (National Institutes of Health Grant 2P400D010949) for the pHD-sfGFPScarlessDsRed vector.

The authors declare no competing financial interests.

Correspondence should be addressed to Craig Montell at cmontell@ucsb.edu.

https://doi.org/10.1523/JNEUROSCI.0200-21.2021

Copyright $\odot 2021$ the authors
}

(Wes et al., 1995; Zhu et al., 1995). Mice encode seven TRPC channels, whereas humans encode only six because human TRPC2 is a pseudogene (Wes et al., 1995; Montell, 2001). Flies and worms each express three TRPC channels (Venkatachalam and Montell, 2007; Xiao and $\mathrm{Xu}, 2009)$. Mammals encode 27 TRPs, which fall into six subfamilies based on amino acid sequence relatedness.

In Drosophila photoreceptor cells, TRP is activated through a signaling cascade that couples light activation of rhodopsin to a G-protein $\left(\mathrm{G}_{\mathrm{q}}\right)$ that stimulates phospholipase C (PLC; Montell, 2012). This leads to hydrolysis of phosphatidylinositol 4,5-bisphosphate $\left(\mathrm{PIP}_{2}\right)$. Wild-type photoreceptor cells display a sustained light response. However, null mutations in trp cause a transient light response. Drosophila TRP has a 40:1 selectivity for $\mathrm{Ca}^{2+}$ relative to $\mathrm{Na}^{+}$, and $\mathrm{Ca}^{2+}$ influx prevents excessive PLC activity, which would otherwise lead to $\mathrm{PIP}_{2}$ depletion (Hardie et al., 2001). In the absence of TRP, the remaining light response is mediated by activation of the nonspecific cation channel, TRPL. The response cannot be maintained because $\mathrm{PIP}_{2}$ is quickly depleted. Coupling to PLC is a common feature among many TRP channels.

Beginning with TRP vanilloid 1 (TRPV1), the structures of many TRP channels have been solved through single-particle 


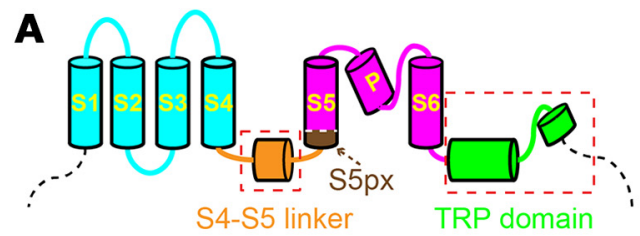

B
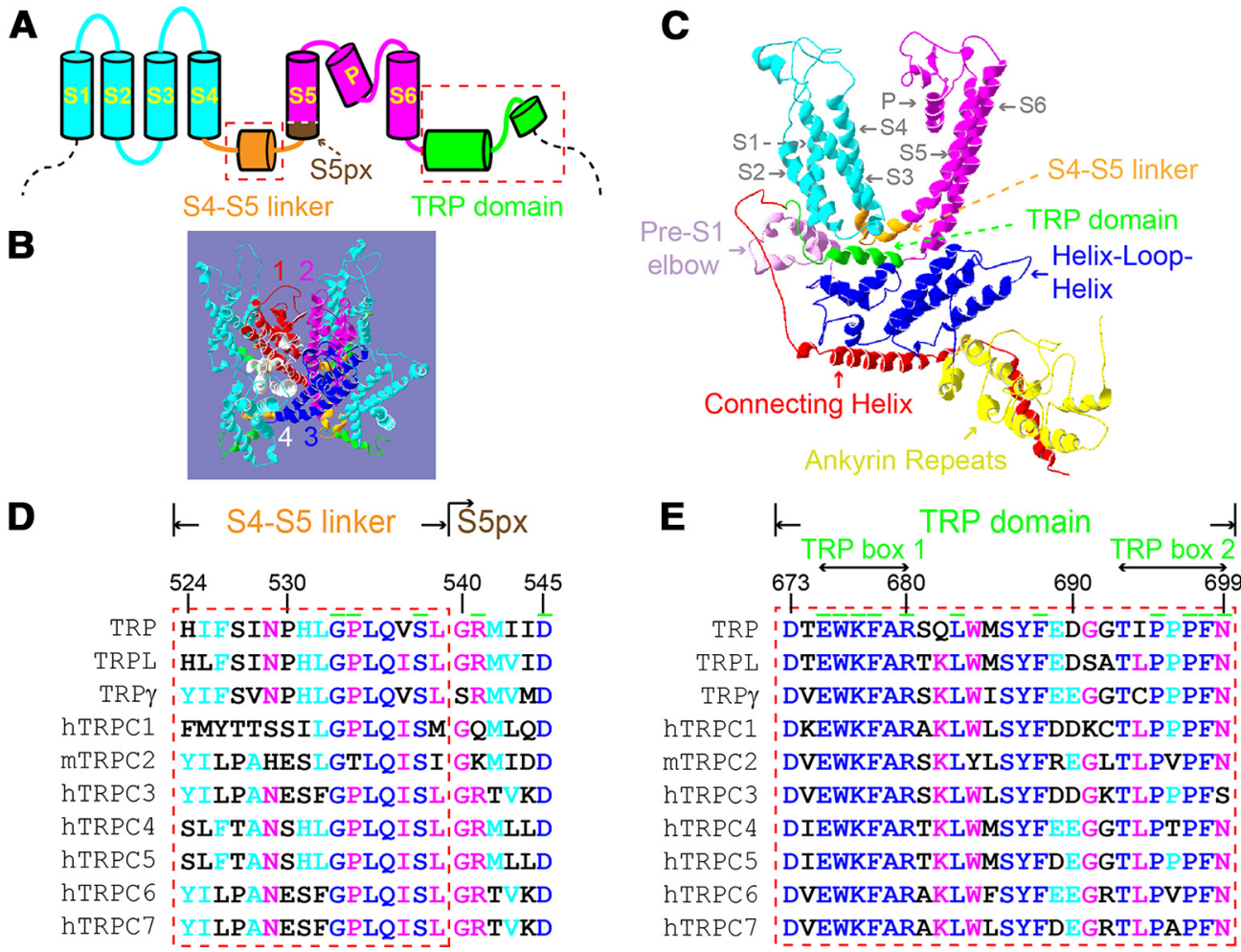

$\mathbf{F}$

$\begin{array}{c:ccc}\text { S4-S5 linker [G533A][P534A][S538A] } & \text { TRP box } 1 & {[\mathrm{E} 675 \mathrm{~A}][\mathrm{W} 676 \mathrm{~A}][\mathrm{K} 677 \mathrm{~A}][\mathrm{F} 678 \mathrm{~A}][\mathrm{R680A}]} \\ \text { S5px } & {[\mathrm{R} 541 \mathrm{~A}][\mathrm{D} 545 \mathrm{~A}]} & \text { TRP box 1-2L } & {[\mathrm{L683A}][\mathrm{F688A}]} \\ & & \text { TRP box } 2 & {[\mathrm{P} 695 \mathrm{~A}][\mathrm{P697A}][\mathrm{F698A}][\mathrm{N} 699 \mathrm{~A}]}\end{array}$

Figure 1. Alanine mutagenesis of the $S 4-S 5$ linker, $S 5 \mathrm{px}$, and the TRP domain. $A$, Diagram depicting the six transmembrane segments (S1-S6); the $S 4-S 5$ linker; the $S 5$-Pore-S6 domain (S5-P-S6); the S5px region, which is the $S 5$ section that is proximal to the $S 4-S 5$ linker; and the TRP domain. $B$, Model of the structure of four Drosophila TRP subunits based on the mouse TRPC4 structure (Duan et al., 2018). The S5-P-S6 domain in each of the four subunits is indicated in (1) red, (2) magenta, (3) blue, and (4) white. C, Ribbon diagram showing key domains in a single TRP subunit. The structure is based on molecular modeling using the structure of mouse TRPC4 as the template (Duan et al., 2018). $\boldsymbol{D}, \boldsymbol{E}$, Alignment of the amino acid sequences of the S4-S5 linker, S5px, and the TRP domain in the Drosophila TRPCs, human TRPC1, 3-7 and mouse TRPC2. Mouse TRPC2 is shown as human TRPC2 is a pseudogene (Wes et al., 1995). Colors indicate different degrees of conservation: blue, $100 \%$ identical; magenta, $80-90 \%$ identical; cyan, $50-70 \%$ identical; black, $<50 \%$ identical. The numbers above the sequences correspond to residues in TRP. Residues mutated by alanine substitutions are indicated with green lines above. $\boldsymbol{F}$, Sixteen residues subjected to alanine (A) mutagenesis. The wild-type amino acid is indicated to the left of the residue number.

electron cryomicroscopy (cryo-EM; Liao et al., 2013; Hilton et al., 2019; Vangeel and Voets, 2019). All have four monomers, each of which is composed of six transmembrane segments (S1S6). A central pore is lined by S5 and S6, and a pore helix (S5-PS6) from four subunits. S1-S4 is situated at the periphery of each monomer, thereby creating a pinwheel-like structure.

S1-S4 and S5-P-S6 in TRP channels are connected by a cytoplasmic $\alpha$-helical S4-S5 linker (Fig. $1 A-C$ ). Movement of this linker is thought to promote gating (Liao et al., 2013; Teng et al., $2015,2016)$. An $\sim 25$ amino acid TRP domain is immediately Cterminal to S6 and runs parallel to the inner leaflet of the membrane. This domain, which includes TRP box 1 (an invariant EWKFAR sequence) and TRP box 2 (typically PPPFN), is conserved in TRPC, TRPM, TRPV, and TRPN channels (Montell, 2001, 2005).

The S4-S5 linker is a hotspot for mutations causing diseases (Hofmann et al., 2017). These include familial episodic pain syndrome (Kremeyer et al., 2010; Zíma et al., 2015), cardiac diseases (Stallmeyer et al., 2012; Xian et al., 2020), skeletal dysplasia, neuropathies (Hofmann et al., 2017), and Olmsted syndrome, which leads to severe itching (Lin et al., 2012; Zhong et al., 2021). The TRP domain in TRPV3 is also a hotspot for mutations causing Olmsted syndrome (Lin et al., 2012; Ni et al., 2016; Zhong et al.,
2021). Mutations in S5 that are proximal to the S4-S5 linker (S5px) cause disease as well (Hofmann et al., 2017).

Because of the importance of the S4-S5 linker, S5px, and the TRP domain for function, we introduced mutations in conserved residues in these domains in Drosophila TRP so that we could determine the functional impact in the native context (photoreceptor cells) using electroretinogram recordings. Based on deficits in the light responses and analyses of protein expression, our study implicates key residues in these regions that are important for channel function, including opening, closing, and protein folding. Our analyses also reveal a functional intrasubunit interaction between the S4-S5 linker and S5px, which contributes to channel function.

\section{Materials and Methods}

Generation of transgenic lines with alanine substitutions in TRP. To create mutations in trp, we introduced them into a genomic construct that included all of the trp introns and the trp promoter. In addition, we fused the sequence encoding the super-folder GFP (sfGFP; green fluorescent protein) in frame with the $5^{\prime}$ end of the trp coding sequence. To generate these constructs, we first used polymerase chain reaction (PCR) amplification of a $0.7 \mathrm{~kb}$ DNA sequence encoding sfGFP from $\mathrm{pHD}$ sfGFP-ScarlessDsRed (catalog \#1365, Drosophila Genomics Resource 
Center) and inserted it after the start codon of $\operatorname{trp}$ within the 6.5-kb trp genomic DNA (Montell et al., 1985). We then subcloned this $\operatorname{trp}$-sfGFP DNA into the pattB vector (Bischof et al., 2007) to create pattB $[s f G F P::$ TRP].

We used PCR-mediated mutagenesis to introduce 16 individual mutations in pattB [sfGFP::TRP], which created alanine substitutions. These included three mutations in the S4-S5 linker (G533A, P534A, S538A), two mutations in S5 proximal to the S4-S5 linker (S5px: R541A and D545A), and 11 in the TRP domain (E675A, W676A, K677A, F678A, R680A, L683A, F688A, P695A, P697A, F698A and N699A).

We generated 17 transgenic lines by integrating the above 16 alanine substitutions as well as a wild-type pattB $[s f G F P:: T R P]$ control into the $\mathrm{ZH}-86 \mathrm{Fb}$ site using PhiC31 integrase-mediated transgenesis (Bischof et al., 2007; BestGene and Rainbow Transgenic Flies). We then removed the white marker gene by crossing each $\operatorname{trp}$ transgene with a genetically encoded Cre recombinase (cata$\log$ \#1501, Bloomington Drosophila Stock Center), and recombined each transgene individually into a $\operatorname{trp} p^{343}$ background.

Western blots. Western blots were conducted essentially as we described previously (Chen et al., 2015). Briefly, proteins were extracted from the heads of flies $1 \mathrm{~d}$ old, fractioned by SDS-PAGE, and transferred to nitrocellulose membranes. After probing with rabbit anti-TRP (Chevesich et al., 1997) and mouse anti-tubulin (catalog \#12G10, Developmental Studies Hybridoma Bank) primary antibodies, we used donkey anti-rabbit IRDye $800 \mathrm{CW}$ (catalog \#926-32213, LI-COR Biosciences) and goat anti-mouse IRDye 680LT (catalog \#926-68020, LI-COR) as the secondary antibodies. We visualized the signals on the Western blots using an Odyssey CLx system, and quantified the relative TRP levels by normalizing using anti-tubulin as an internal loading control. The quantification results were based on three replicates from independent protein samples.

Electroretinogram recordings. We performed electroretinogram (ERG) recordings as described (Wes et al., 1999). Flies (1 d after eclosion) were exposed to $10 \mathrm{~s}$ pulses of bright orange light $\left(\sim 30 \mathrm{~mW} / \mathrm{cm}^{2}\right)$, and $\geq 6$ flies were tested for each genotype. To quantify the transient ERG phenotype, we determined the time required for a $60 \%$ decline from the peak light-induced amplitude back to the baseline ( $t_{60}$ deactivation; Wang et al., 2005; Chen et al., 2015). To quantify the slow deactivation phenotype displayed by $\operatorname{trp}{ }^{S 538 A}$ flies, we determined the time required for a $50 \%$ return to the baseline after cessation of the light stimulus ( $t_{50}$ termination). For those flies that showed a sustained ERG response during the light stimulus, we quantified the amplitude of the sustained response $(\mathrm{mV})$ at the end of $10 \mathrm{~s}$ light stimulus.

Immunostaining of whole-mounts of compound eyes. Immunostaining of whole-mounts of compound eyes was performed essentially as described (Chen et al., 2015). Briefly, we fixed the tissue for $2 \mathrm{~h}$ on ice in PBS and $4 \%$ paraformaldehyde, dissected out the eyes in PBS and

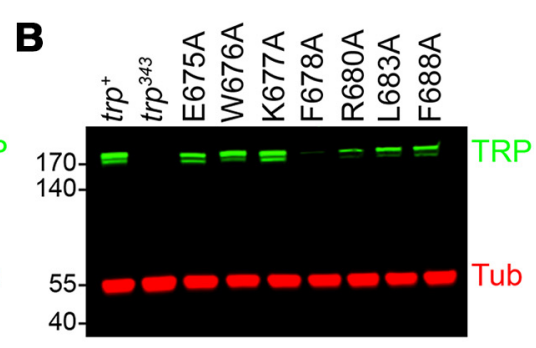

D

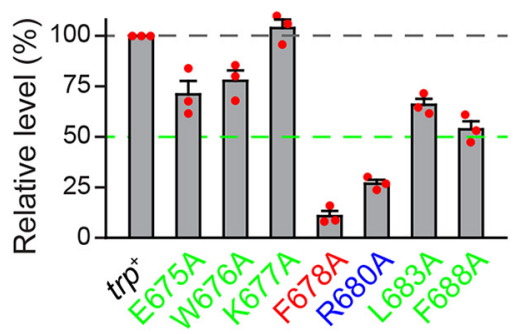

G

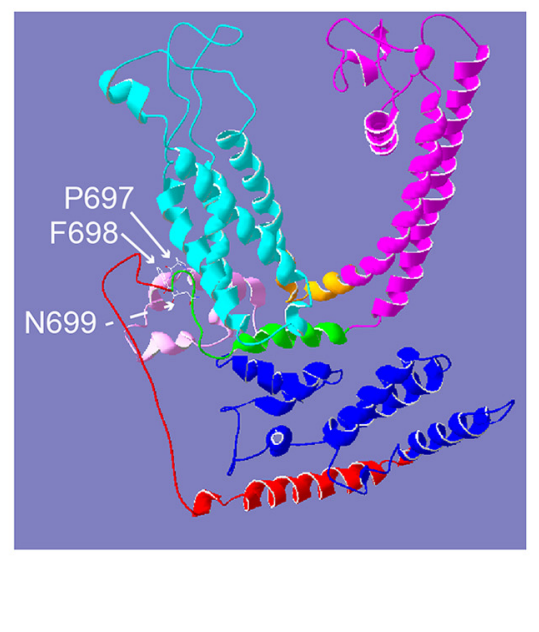

Figure 2. TRP expression levels and modeling of the region including PFN in TRP box 2. $A$, Western blot showing expression of TRP containing mutations in the S4-S5 linker and S5px. Head extracts were fractionated by SDS-PAGE and probed with rab年 $(\mathrm{kDa})$. $\boldsymbol{B}$, Western blot showing expression of TRP containing mutations , whereas blue indicates expression of $\sim 25 \%$. $\boldsymbol{E}$, Western blot showing expression of TRP containing mutations Ribbon diagram indicating the positions and side chains of residue P697, F698, and N699. $n=3$. Means \pm SEM.

$0.1 \%$ Triton X-100, and incubated the samples for two nights at $4^{\circ}$ C with mouse anti-Rh1 (catalog \#4C5, Developmental Studies Hybridoma Bank) and chicken anti-GFP (catalog \# A-10262, Invitrogen), which recognizes the sfGFP::TRP fusion protein. We then washed for 20 min three times in PBS and $0.1 \%$ Triton X-100, and incubated the samples with Alexa Fluor 488 anti-chicken (catalog \#A-11039, Invitrogen) and Alexa Fluor 568 anti-mouse (cata$\log$ \#A-11004, Invitrogen) secondary antibodies. We acquired $1 \mu \mathrm{m}$ optical sections of the compound eye at the distal R7 cell level using a Zeiss LSM 700 confocal microscope.

SWISS-MODEL of TRP structure. We used SWISS-MODEL (Waterhouse et al., 2018) to build a three dimensional (3D) structure of Drosophila TRP, based on the 5Z96 structural template of mouse TRPC4 (Duan et al., 2018). A PDB viewer was used to show the modeling images. 
Statistical analyses. The bar plots represent means \pm SEM. Individual data points are shown as red dots. We used one-way ANOVA with Holm-Sidak post hoc analyses for multiple comparisons. Statistically significant differences are indicated by asterisks $\left({ }^{*} p<\right.$ $\left.0.05,{ }^{* *} p<0.01\right)$.

\section{Results}

Mutagenesis of the S4-S5 linker, S5px, and the TRP domain To reveal residues in the critical S4-S5 linker, S5px, and the TRP domain required for function of Drosophila TRP in photoreceptor cells, we substituted the nonpolar residue alanine for highly conserved residues in these domains. We targeted three residues in the S4-S5 linker including glycine 533 (Fig. 1D), which is invariant among Drosophila and mammalian TRPC, TRPV, TRPM, and TRPA 1 channels. We also changed the adjacent proline (residue 534) and serine 538, which are highly conserved in TRPC channels (Fig. 1D). In addition, we replaced two charged residues (arginine 541 and aspartic acid 545) in the S5px region (Fig. 1D).

We also introduced 11 single alanine substitutions in the TRP domain. These include five of six amino acids in TRP box 1 (Montell, 2005) except for residue 679, which is an alanine in wild-type TRP, as well as four highly conserved residues in TRP box 2 (Fig. $1 E$ ). Finally, we mutated two residues in the linker between TRP boxes 1 and 2 (TRP box 1-2L), which are invariant among all TRPC channels (leucine 683 and phenylalanine 688; Fig. 1E,F). We introduced each of the 16 trp mutations individually in a $\operatorname{trp}$ genomic transgene so that each trp mutation would be expressed under the normal trp transcriptional control. In addition, each of these proteins, including a wild-type TRP control, was fused to an N-terminal GFP tag. We inserted the transgenes into the same chromosomal site and recombined them individually into the $\operatorname{trp}^{343}$ null background (Montell and Rubin, 1989; Wang et al., 2005).

\section{Residues in S4-S5 linker, S5px, and TRP domain essential for expression}

To determine the impact of the mutations on expression, we prepared head extracts and performed Western blot analysis with anti-TRP, which works effectively on membrane blots. Six of the 16 mutations resulted in low expression levels $(<10 \%$; Fig. $2 A-F$; Table 1). The most profound reduction was caused by the G533A mutation in the S4-S5 linker (Fig. 2A,C), which disrupts a residue that is invariant in all TRPC, TRPV, TRPM, and TRPA1 proteins (Hofmann et al., 2017) and is required for flexibility of the S4-S5 linker (Romero-Romero et al., 2017). In addition, a mutation in the S5px region, D545A, resulted in a dramatic decrease in TRP ( $<5 \%$; Fig. $2 A, C)$. This aspartic acid residue is conserved in all TRPC and TRPV channels in flies and mammals. These findings suggest that these G533 and D545 in the S4-S5 linker and the S5px region are essential for folding of Drosophila TRP.

Four mutations in the TRP domain also greatly reduced protein expression to $<10 \%$. These include F678A, which is within TRP box 1, and three mutations in TRP box 2: P697A, F698A, and N699A. We built a 3D model of TRP using SWISS-MODEL (Waterhouse et al., 2018) based on the 5Z96 template of mouse TRPC4 (Duan et al., 2018). This model indicates that these three residues (P697, F698, and N699) are situated at a radical turn (Fig. 2G), suggesting that this turn is essential for TRP to form a correct conformation.

Nine of the other 16 mutant proteins were expressed at $\geq 50 \%$ of the normal TRP levels (Fig. $2 A-F$ ). These mutations altered
Table 1. Summary of trp point mutations

\begin{tabular}{|c|c|c|c|c|}
\hline Mutation & $\begin{array}{l}\text { Protein } \\
\text { level \% }\end{array}$ & Domain & $\begin{array}{l}\text { Phenotypic } \\
\text { class* }\end{array}$ & ERG phenotype \\
\hline G533A & $<10$ & S4-S5 linker & & transient: similar to null \\
\hline P534A & $\geq 50$ & S4-S5 linker & 2 & transient: not as strong as null \\
\hline S538A & $\geq 50$ & S4-S5 linker & 4 & slow deactivation \\
\hline R541A & $\geq 50$ & S5px & 1 dominant & transient: similar to null but dominant \\
\hline D545A & $<10$ & S5px & & similar to null \\
\hline $\mathrm{E} 675 \mathrm{~A}$ & $\geq 50$ & TRP box 1 & 2 & transient: not as strong as null \\
\hline W676A & $\geq 50$ & TRP box 1 & wild type & wild type \\
\hline K677A & $\geq 50$ & TRP box 1 & 3 & mildly transient \\
\hline F678A & $<10$ & TRP box 1 & & similar to null \\
\hline R680A & 25 & TRP box 1 & 1 & transient: similar to null but mislocalized \\
\hline L683A & $\geq 50$ & TRP box 1-2L & 1 & transient: similar to null \\
\hline F688A & $\geq 50$ & TRP box 1-2L & 2 & transient: not as strong as null \\
\hline P695A & $\geq 50$ & TRP box 2 & wild type & wild type \\
\hline P697A & $<10$ & TRP box 2 & & transient: similar to null \\
\hline F698A & $<10$ & TRP box 2 & & transient: similar to null \\
\hline N699A & $<10$ & TRP box 2 & & transient: not as strong as null \\
\hline
\end{tabular}

*Phenotypic classes apply only to mutations with TRP levels $\geq 25 \%$.

residues in the S4-S5 linker (P534A and S538A), the S5px region (R541A), TRP box 1 (E675A, W676A, K677A) or TRP box 1-2L (L683A, F688A). In addition, a 10th mutation, R680A, which changed a residue in TRP box 1, resulted in expression at $\sim 25 \%$ normal levels (Fig. $2 B, D$ ). The levels of these 10 proteins are significant because we showed earlier that expression of wild-type TRP at $10 \%$ of the normal concentration is sufficient to produce a normal light response (Wang et al., 2005). Thus, if any of these latter 10 mutations affect channel function it would not be a consequence of reduced expression.

\section{S4-S5 linker and TRP domain are critical for function}

In contrast to most TRPC channels, Drosophila TRP has been very difficult to functionally express in tissue culture cells or Xenopus oocytes because it fails to exit the endoplasmic reticulum. However, the advantage of a mutational analysis of Drosophila TRP is that it provides the opportunity to assess the effects of amino acid substitutions on function in its native system-photoreceptor cells. To characterize the light response in intact flies we used ERG recordings. This field recording is performed by placing a recording electrode on the surface of the fly eye and measuring the light response of the retinal cells within the conduction field near the electrode. In wild-type controls, a $10 \mathrm{~s}$ light pulse induces a sustained response during the light stimulation (Fig. $3 A, U$ ). After cessation of the light response, the sustained component quickly declines (Fig. $3 A, C$ ). To assess the rate of termination of the photoresponse, we measured the time (s) required for a $50 \%$ return to the baseline $\left(t_{50}\right.$; Fig. $\left.3 \mathrm{~V}\right)$. The $t_{50}$ is more accurate than determining the full return to the baseline after cessation of the light as there is often a slight overshoot (Fig. 3C), rendering it difficult to accurately determine precisely the time for a complete return. The on and off transients near the initiation and coincident with the cessation of the light pulse are because of activity postsynaptic to the photoreceptor cells in the optic lobes (Fig. $3 A$ ). Loss of TRP such as in the $\operatorname{trp}^{343}$ null allele (Wang et al., 2005) leads to a transient ERG response (Fig. 3B; Cosens and Manning, 1969). Fusion of GFP to wild-type TRP does not impair function as expression of GFP::TRP in a $t r p^{343}$ background fully rescues the transient ERG phenotype (Fig. 3C). 


\section{A \\ B \\ C \\ D $\quad$ E \\ F \\ G}
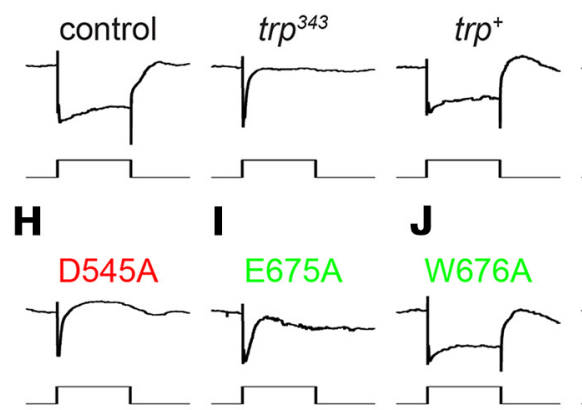

G533A

E

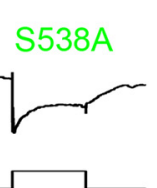

R541A
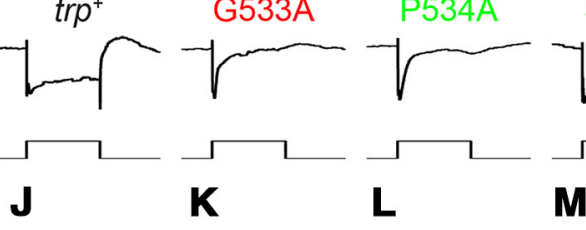

M N
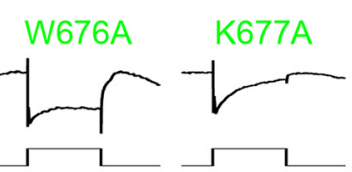

F678A

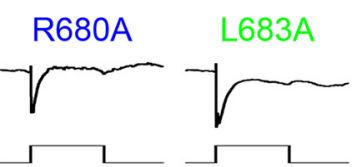

o

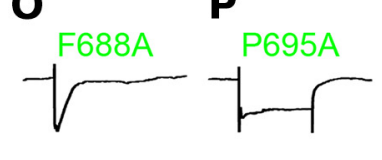

Q $\quad$ R

R S
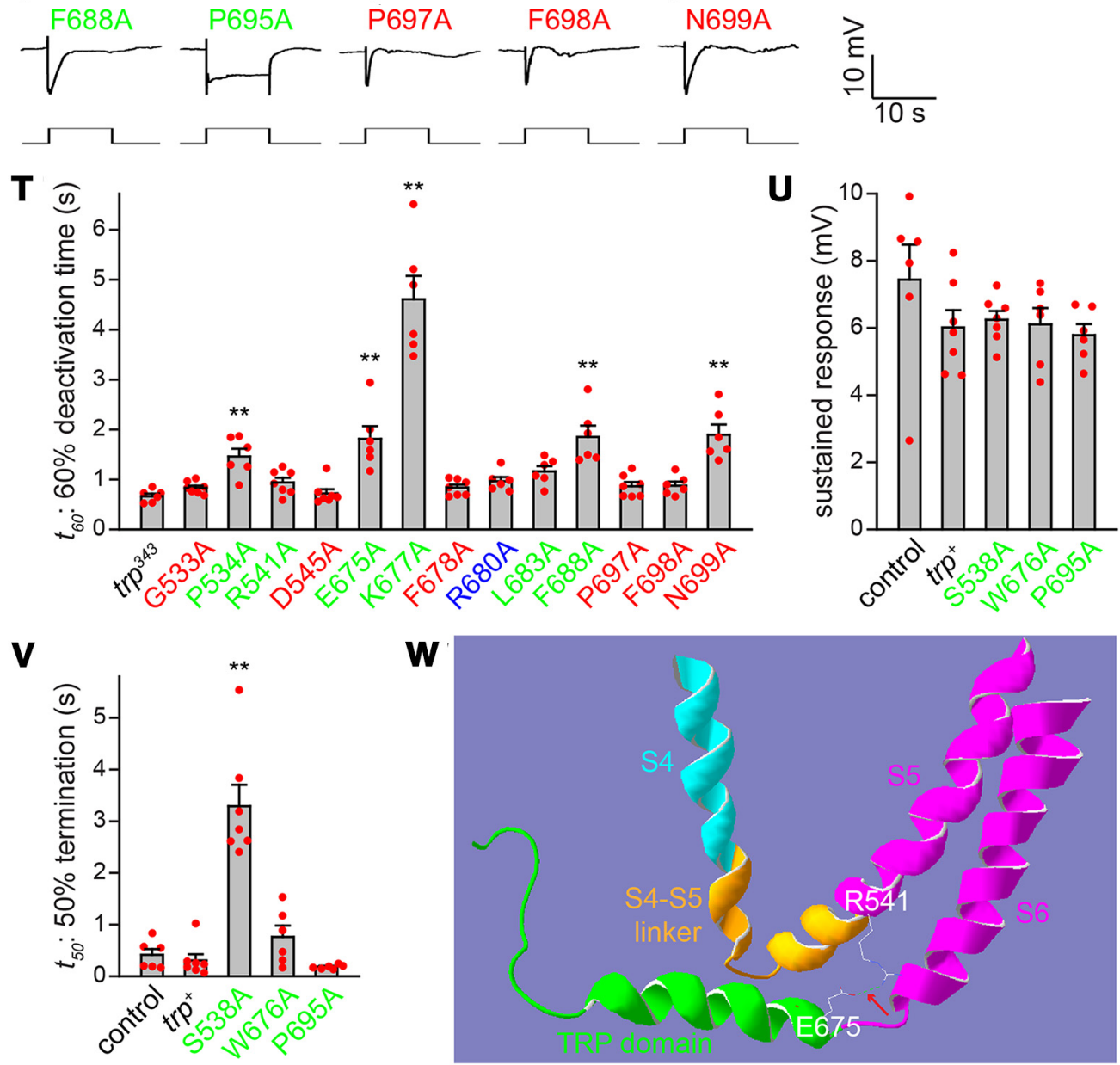

Figure 3. Effects of trp mutations on the light response using ERGs. $\boldsymbol{A}-\mathbf{S}$, Shown are representative ERG traces from the indicated genotypes. Flies were stimulated with a $10 \mathrm{~s}$ pulse of light indicated below each trace. $\boldsymbol{A}$, Control $\left(w^{1718}\right) ; \boldsymbol{B}, \operatorname{trp}^{343} ; \boldsymbol{C}, \operatorname{trp}^{+}$transgene; $\boldsymbol{D}, \operatorname{trp}^{6533 A} ; \boldsymbol{E}, \operatorname{trp}^{P 534 A} ; \boldsymbol{F}, \operatorname{trp}^{5538 A} ; \boldsymbol{G}, \operatorname{trp}^{R 541 A} ; \boldsymbol{H}, \operatorname{trp}^{0545 A} ; \boldsymbol{I}, \operatorname{trp}^{6675 A} ; \boldsymbol{J}, \operatorname{trp}^{W 676 A} ; \boldsymbol{K}, \operatorname{trp}^{K 677 A} ; \boldsymbol{L}, \operatorname{trp}^{\text {G678A }} ; \boldsymbol{M}, \operatorname{trp}^{R 680 A} ;$

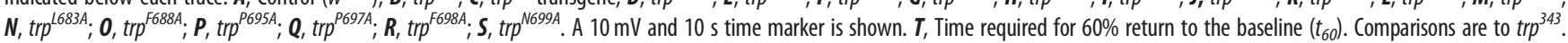
$\boldsymbol{U}$, Sustained ERG depolarization $(\mathrm{mV})$ at the end of the $10 \mathrm{~s}$ light pulse stimulus. $\boldsymbol{V}$, Time required for $50 \%$ return of the ERG response after cessation of the light stimulus $\left(t_{50}\right)$. $\boldsymbol{W}$, Ribbon diagram showing the hydrogen bond (indicated by the arrow) between R541 in the S5px and E675 in TRP box 1. $n \geq 6$. Means \pm SEM. One-way ANOVA with Holm-Sidak post hoc analyses. **, $p<0.01$.

We used ERGs to compare the impact of the various mutations on the light response. For those mutants that are expressed at high levels and are localized normally in the rhabdomeres (see below, Spatial localizations of mutated TRP proteins), then a decline in the sustained component during the light response is indicative of an impairment in channel activity-either in opening of the TRP channels or in remaining open. If the sustained component is maintained (channels remain open during the light stimulus), but there is a slow termination after cessation of the light stimulus, then this suggests a defect in closing of the TRP channels. Consistent with their large reductions in TRP levels $(<10 \%)$, the G533A, D545A, F678A, P697A, F698A, and N699A mutations caused transient ERGs (Fig. 3D,H,L,Q-S). To assess whether these light responses decayed as quickly as in $t r p^{343}$, we determined the time that it took for the ERG responses to decline back to baseline by $60 \%\left(t_{60}\right)$-a parameter that we have used previously to compare the severities of different trp alleles (Wang et al., 2005; Chen et al., 2015). The $t_{60}$ for $t r p^{343}$ is $0.66 \pm 0.05 \mathrm{~s}$, 
A
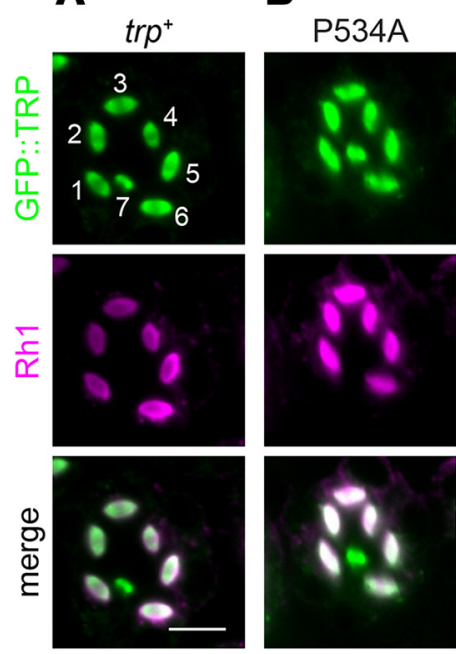

G R680A
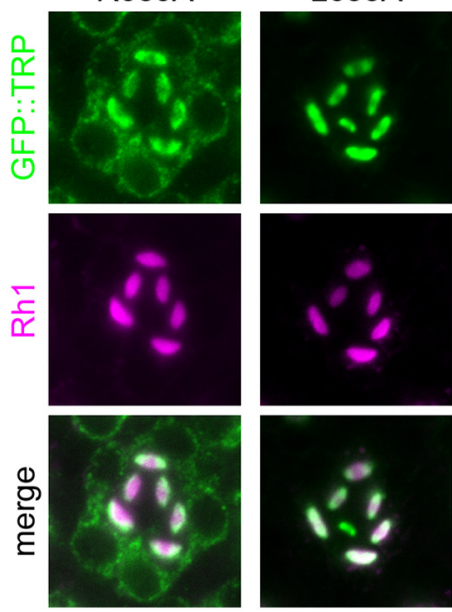

B

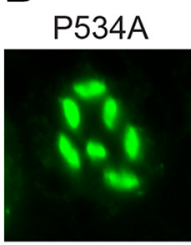

C
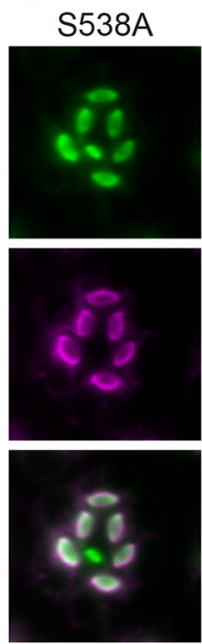

H

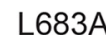

I F688A
D
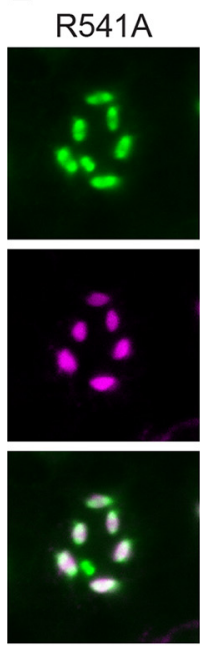

J
E
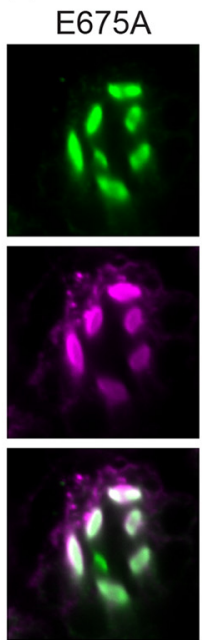

F K677A
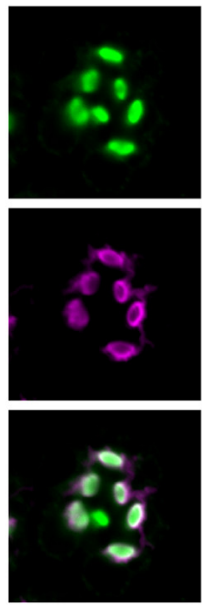
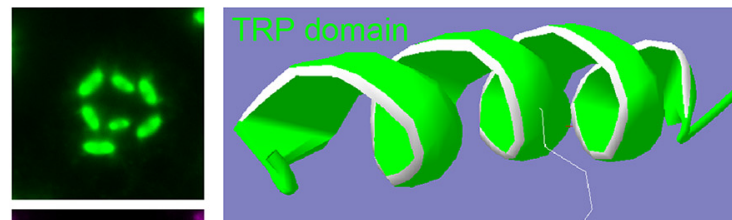

R680
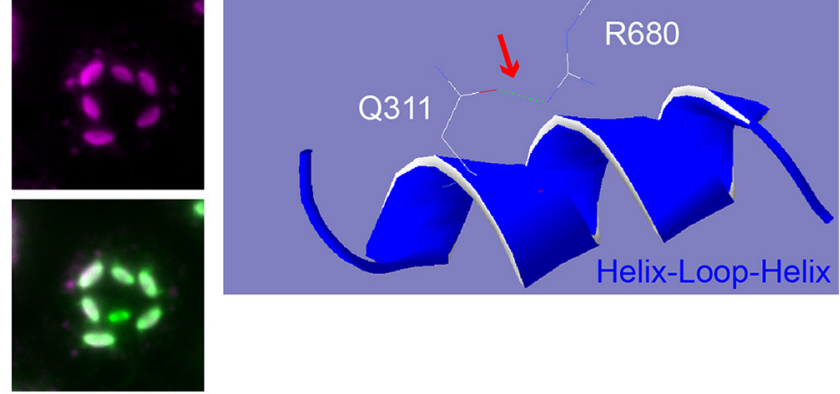

Figure 4. Spatial localization of mutated TRP channels in photoreceptor cells. A-I, All of the TRP isoforms were fused to GFP and introduced into a trp ${ }^{343}$ mutant background. Whole mounts of compound eyes were stained with anti-GFP (green, top rows) and anti-Rh1 (magenta, middle rows), and optical cross-sections of individual ommatidia are shown at the distal regions, which include the R1-6 and R7 cells. Wild type GFP::TRP is localized to the rhabdomeres of all photoreceptor cells, whereas Rh1 is localized to the rhabdomeres of the R1-6 cells. The merge of the anti-GFP and anti-Rh1 staining is shown in the bottom rows. The compound eyes were dissected and stained from the indicated flies. $\boldsymbol{A}$, Wild-type trp ${ }^{+}$transgene (sfGFP::TRP). Scale bar,

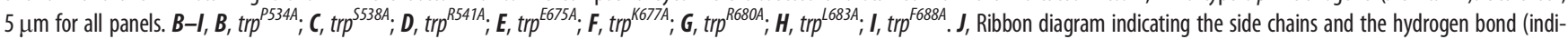
cated by the arrow) between residues R680, which is localized to the TRP domain, and Q311, which is in the helix-loop-helix.

and most of these mutations resulted in similar transient responses (Fig. 3D,H,L,Q,R,T). However, the $t_{60}$ for N699A was slightly longer, suggesting that it is a hypomorphic allele that maintains residual TRP channel activity $(1.9 \pm 0.2 \mathrm{~s}$; Fig. 3S,T).

The ERG phenotypes of the nine mutant TRPs that were expressed at high levels ( $\geq 50 \%)$ were of particular interest as any alteration in the ERG response would not simply be because of low expression. In addition, the 10th mutant protein (R680A) was expressed at $25 \%$, which is also above the $10 \%$ threshold needed for function of wild-type TRP. Two mutations that were located in TRP box 1 (W676A) and TRP box 2 (P695A) exhibited sustained responses during light stimulation and times for $50 \%$ decline in the sustained component of the ERG after cessation of the light $\left(t_{50}\right)$ that were indistinguishable from the $t r p^{+}$ control (Fig. 3J,P, $U, V$ ). This indicates that these two residues are tolerant to alanine substitutions. The remaining eight mutations caused abnormal ERG responses, demonstrating their importance for function. The ERG phenotypes varied. R541A (S5px),
R680A (TRP box 1), and L683A (linker between TRP boxes 1 and 2; TRP box 1-2L) caused the severest ERG phenotypes (class 1 ; Table 1 ) as the $t_{60}$ times were not significantly different from $\operatorname{trp}^{343}$ (Fig. 3G,M,N,T). Thus, these three mutations eliminated TRP function. The P534A (S4-S5 linker), E675A (TRP box 1), and F688A (TRP box 1-2L) mutations resulted in marginally weaker transient ERG phenotypes (class 2; Table 1) with slightly longer $t_{60}$ times than the null mutant (Fig. 3E,I,O,T), indicating that these mutants still retained some residual TRP channel activity. Based on structural modeling of Drosophila TRP, E675, which is near the beginning of TRP box 1, forms a hydrogen bond with R541, which is positioned right after the S4-S5 linker of the same subunit (Fig. $3 W$ ). The class 3 phenotype included K677A (TRP box 1) and was characterized by a transient ERG phenotype with a much slower decay indicating that it is a hypomorphic mutation (Fig. $3 K, T ; t_{60}=4.6 \pm 0.5 \mathrm{~s}$; Table 1). Finally, the main aspect of the class 4 phenotype, which was exhibited by S538A (S4-S5 linker), was slower deactivation kinetics on cessation of the light stimulus (Fig. $3 F, V$; Table 1). However, this 
mutation did not have an impact on the amplitude of the sustained response during the light stimulus (Fig. 3U).

\section{Spatial localizations of mutated TRP proteins}

The ERG impairments resulting from the eight mutations that were expressed $\geq 25 \%$ suggest that the mutations alter channel function. Alternatively, the phenotypes could be because of an impairment in subcellular localization. Wild-type TRP is spatially restricted to the microvillar portion of the photoreceptor cells-the rhabdomeres, where phototransduction takes place (Montell and Rubin, 1989). Therefore, if a mutation caused a trafficking defect that prevented localization in the rhabdomeres, then TRP could not participate in phototransduction. The fly compound eye is composed of $\sim 800$ repeat unitsommatidia-each of which contains eight photoreceptor cells and several other cell types. Six of the photoreceptor cells (R1-6) extend the full depth of the retina and express the major rhodopsin, Rh1. The R7 and R8 photoreceptor cells occupy the distal and proximal regions of the retina, respectively.

To spatially localize the mutant TRP proteins, we took advantage of the GFP tag that we fused to the $\mathrm{N}$ terminus to the transgenic proteins as anti-GFP works very effectively for immunostaining of photoreceptor cells. We stained photoreceptor cells with anti-GFP to visualize TRP and anti$\mathrm{Rh} 1$ as a marker for the R1-6 rhabdomeres. Wild-type GFP::TRP was restricted to the rhabdomeres (Fig. $4 A$ ), a pattern indistinguishable from untagged TRP. Thus, GFP fused to the $\mathrm{N}$ terminus of TRP does not impair trafficking to the rhabdomeres in vivo, consistent with the normal ERG (Fig. 3C). All seven of the mutant TRPs that were expressed at levels $\geq 50 \%$ and that resulted in mutant phenotypes (classes 1, 2, 3 and 4; Table 1) were localized exclusively in the rhabdomeres and colocalized with Rh1 in R1R6 cells (Fig. 4B-F,H-I). Therefore, the mutant phenotypes were not because of defects in TRP trafficking. Thus, all TRPs that were stably expressed ( $\geq 50 \%$ wild-type levels) but caused a mutant phenotype were localized normally. This set of mutant TRPs that are stably expressed are collectively referred to as TRP ${ }^{\mathrm{MSN}}$ [mutant phenotype, stably expressed, and normally localized (MSN)] proteins.

In contrast to the seven $\mathrm{TRP}^{\mathrm{MSN}}$ proteins, the $\mathrm{TRP}^{\mathrm{R} 680 \mathrm{~A}}$ protein is expressed at a lower level (25\% relative to wild type), and a significant proportion is mislocalized to the extrarhabdomeral cell bodies (Fig. 4G). The 3D model of the TRP structure Fig. $4 J$ indicates that R680 in the TRP domain forms a hydrogen bond with a glutamine (residue 311 ), 13 amino acids $\mathrm{N}$ terminal
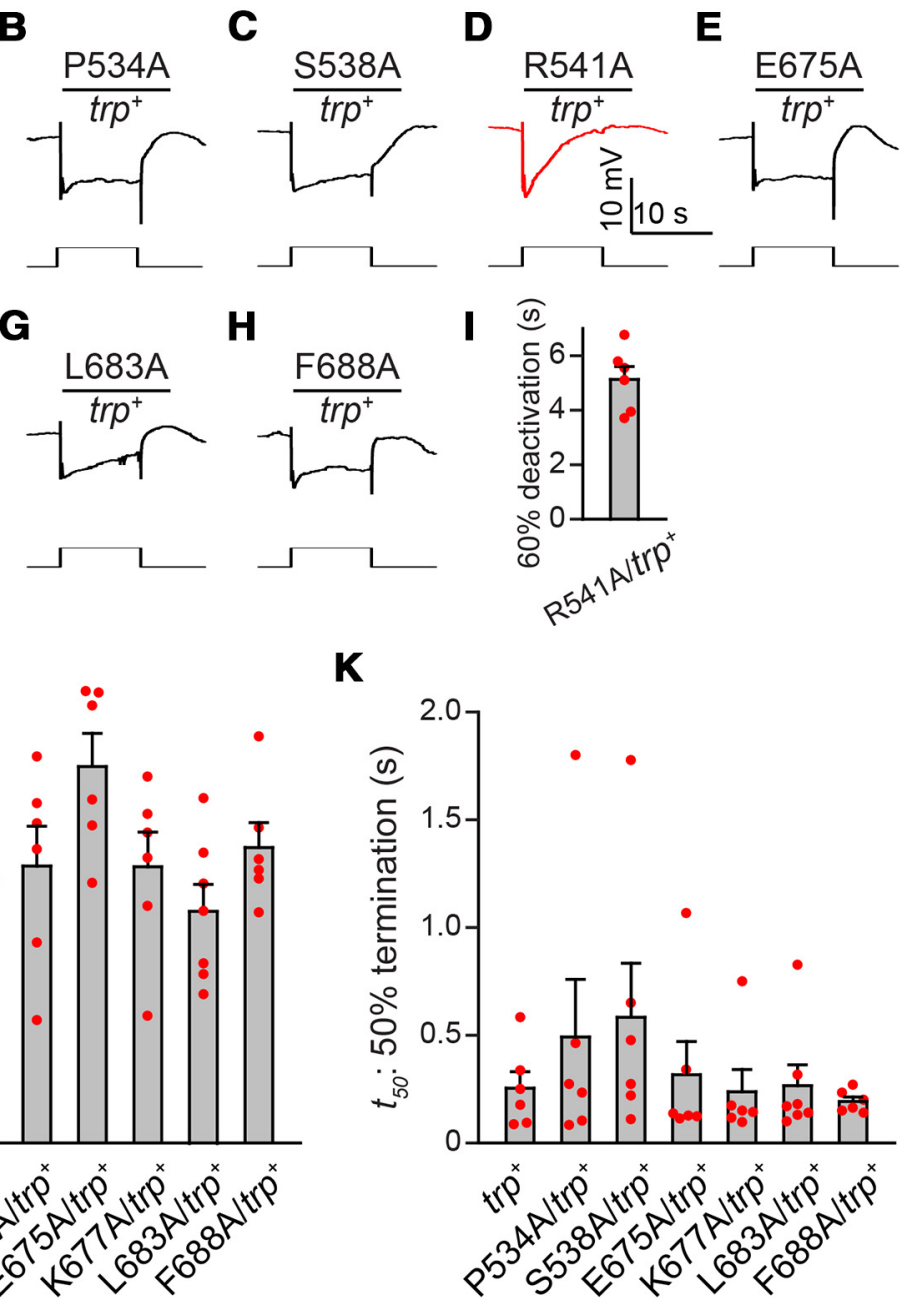

Figure 5. Testing trp mutants for dominant ERG phenotypes. $\boldsymbol{A}$, Flies contained two copies of wild-type transgene (trp $\left.{ }^{+}\right)$in a wild-type transgene $\left(\operatorname{trp}^{+}\right)$in a $\operatorname{trp}^{343}$ background. $\boldsymbol{B}, \operatorname{trp}^{\text {P534A }} ; \boldsymbol{C}, \operatorname{trp}^{S 538 A} ; \boldsymbol{D}, \operatorname{trp}^{R 541 A} ; \boldsymbol{E}, \operatorname{trp}^{E 675 A} ; \boldsymbol{F}, \operatorname{trp}^{K 677 A} ; \boldsymbol{G}, \operatorname{trp}^{\text {L683A }} ; \boldsymbol{H}$, $\operatorname{trp}^{F 688 A}$. Flies were stimulated with a $10 \mathrm{~s}$ pulse of light indicated below each trace. I, Time required for the $\operatorname{trp}^{R 541 A} / \operatorname{trp}^{+}$ERG to display a $60 \%$ return to the baseline $\left(t_{60}\right)$. $\boldsymbol{J}$, Amplitude of sustained ERG responses at the end of the $10 \mathrm{~s}$ light stimulus. $\boldsymbol{K}$, Time required for $50 \%$ return of the ERG response after cessation of the light stimulus $\left(t_{50}\right) . n \geq 6$. Means \pm SEM.

to pre-S1 helix (Wang et al., 2020). Residues corresponding to R680 and Q311 are invariant in the other fly and mammalian TRPC channels. The interaction among these highly conserved residues in the $\mathrm{N}$ - and $\mathrm{C}$-terminal ends of TRP would appear to be important for protein folding, which has an impact on trafficking to the rhabdomeres.

Interallelic suppression of a dominant-negative phenotype TRP channels are tetramers. Therefore, we tested whether any of the TRP ${ }^{\mathrm{MSN}}$ proteins caused a dominant phenotype in photoreceptor cells. We performed ERGs on the seven $\operatorname{trp}^{M S N} /+$ heterozygous flies and found that most displayed wild-type ERGs (Fig. $5 A-C, E-H, J, K$ ). However, $\operatorname{trp} p^{R 541 A} /+$ heterozygous flies exhibited a transient ERG phenotype $\left(t_{60}=5.1 \pm 0.5 \mathrm{~s}\right.$; Fig. $\left.5 D, I\right)$, demonstrating that incorporation of the R541A subunit with wild-type subunits is sufficient to disrupt the TRP tetramer and create a dominant-negative phenotype. The R541 residue is located in the S5px region (Fig. 1D), and forms a hydrogen bond with E675, which may contribute to promoting the interface between S4-S5 linker and TRP domain (Fig. $3 W$ ). 


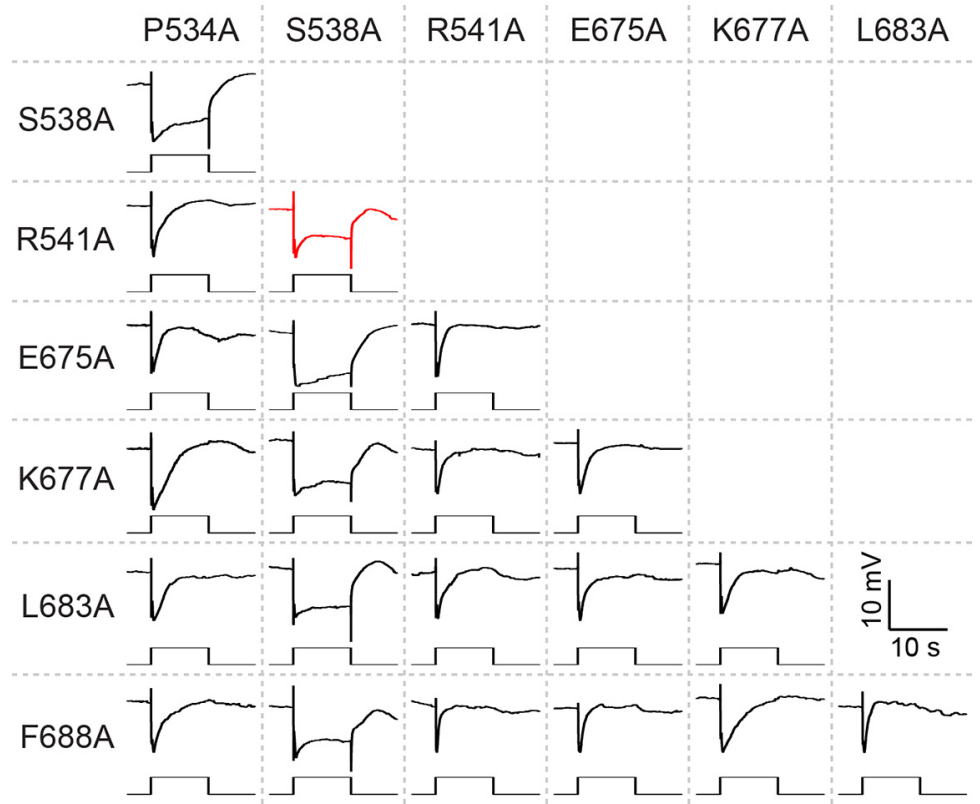

Figure 6. Suppression of trp ERG phenotypes in trans-heterozygous flies. Top row and left column indicate the two corresponding mutations analyzed in the trans-heterozygous combinations. Flies were stimulated with a $10 \mathrm{~s}$ pulse of light indicated below each trace. The ERG traces are shown in the corresponding boxes.

We then tested for interallelic complementation to determine whether a tetramer comprising two mutant $\mathrm{TRP}^{\mathrm{MSN}}$ subunits would suppress each other and result in a wild-type ERG. Six of the $\operatorname{trp}{ }^{M S N}$ alleles elicit transient ERG phenotypes as homozygous flies including $\operatorname{trp}{ }^{R 541 A}$, which is a dominant-negative allele. We found that trans-heterozygous combinations of any of these six alleles displayed transient ERG responses (Figs. 6, $7 A ; \operatorname{tr} p^{P 534 A}$, $\operatorname{tr} p^{R 541 A}, \operatorname{tr} p^{E 675 A}, \operatorname{trp}{ }^{K 677 A}, \operatorname{tr} p^{L 683 A}$ and $\left.\operatorname{tr} p^{F 688 A}\right)$, demonstrating that there was little if any interallelic complementation.

We also tested for interallelic complementation among the six alleles with transient ERG phenotypes and $\operatorname{trp}^{S 538 A}$, which is distinct from the other $\operatorname{tr} p^{M S N}$ alleles in that it displays a slow deactivation phenotype. We found that trans-heterozygous combinations between $\operatorname{tr} p^{S 538 A}$ and any of the other six $\operatorname{trp}{ }^{M S N}$ alleles resulted in wild-type ERGs (Figs. 6, 7B,C). Most strikingly, the $\operatorname{tr} \mathrm{S}^{S 538 \mathrm{~A}} / \operatorname{tr} p^{R 541 A}$ flies exhibited a normal ERG (Fig. 6), although $\operatorname{tr} p^{R 541 A} /+$ flies display a transient ERG phenotype. We examined the structural model for TRP and found that the R541 backbone forms a predicted hydrogen bond with the residue adjacent to S538 (valine 537), whereas the side chain of S538 forms a hydrogen bond with L535, which is an invariant residue among all fly and mammalian TRPC channels (Figs. 1D, 7D).

\section{Discussion}

\section{Distinct effects of S4-S5 linker mutations on TRP opening and closing in vivo}

Mutations in the S4-S5 linker can lead to constitutive activity of TRP channels in vitro, including TRPC4 and TRPC5 (Beck et al., 2013). We found that mutations in the S4-S5 linker of Drosophila TRP result in different types of deficits in photoreceptor cells, indicating that distinct substitutions in the S4-S5 linker can affect either the light-dependent opening of TRP or rapid closing following cessation of light. In support of this conclusion, mutation of proline 534 caused a transient ERG, whereas mutation of serine 538 resulted in slow deactivation on termination of light. These phenotypes were not because of low expression or impairments in trafficking to the rhabdomeres as TRP ${ }^{\mathrm{P} 534 \mathrm{~A}}$ and
$\mathrm{TRP}^{\mathrm{S} 538 \mathrm{~A}}$ were localized specifically in the rhabdomeres and expressed at normal levels (TRP ${ }^{\mathrm{MSN}}$ proteins). Our findings that mutations in the S4-S5 linker lead either to a failure in channel opening $\left(\mathrm{TRP}^{\mathrm{P} 534 \mathrm{~A}}\right)$ or slow deactivation $\left(\mathrm{TRP}^{\mathrm{S} 538 \mathrm{~A}}\right)$ following light cessation support the model that movement of the S4-S5 linker promotes channel opening (Liao et al., 2013; Teng et al., 2015, 2016) and that different residues within this region contribute to the opening or closing of TRP. The large effect of the P534A mutation on activation may be because of decreased flexibility of the S4-S5 linker because it is part of a glycine-proline dipeptide that appears to be necessary for linker flexibility (Romero-Romero et al., 2017). A local hydrogen bond between serine 538 and leucine 535, which are both invariant in fly and mammalian TRPCs, may contribute to the stiffness of the S4-S5 linker. The S538A may cause the S4-S5 linker to become more flexible, thereby increasing the time necessary for channel closing. The slow inactivation resulting from the $\mathrm{TRP}^{\mathrm{S} 538 \mathrm{~A}}$ mutation is distinct from the effects resulting from S4-S5 linker mutations in human TRPs (Hofmann et al., 2017). Given the conservation of the serine in TRPC channels, it would be intriguing to investigate whether mutation of this residue in human TRPCs also causes slow deactivation.

Mutations in the S4-S5 linker of TRPs are associated with disease, and the clinical manifestations appear to be caused by constitutive channel activity (Hofmann et al., 2017). An example is Olmstead syndrome, which results from mutation of glycine 573 in TRPV3 (Xiao et al., 2008; Lai-Cheong et al., 2012; Lin et al., 2012). This glycine in the S4-S5 linker is conserved in all TRPC, TRPA, TRPV, TRPM, and TRPN channels. Mutation of the corresponding glycine in Drosophila TRP $\left(\mathrm{TRP}^{\mathrm{G} 533 \mathrm{~A}}\right)$ appears to be critical for protein folding as $\mathrm{TRP}^{\mathrm{G} 533 \mathrm{~A}}$ is undetectable, which is presumably because of degradation of the misfolded protein. Thus, the S4-S5 linker appears to be required for activation and protein folding in photoreceptor cells.

\section{Activation affected by an intersubunit interaction between S4-S5 linker and S5}

In contrast to most disease mutations in human TRPs, which increase channel activity or cause constitutive activity, none of the mutations in the highly conserved residues in Drosophila TRP result in constitutive activity. However, a mutation in S5 of Drosophila TRP (F550I) is a gain-of-function mutation with constitutive activity, which leads to severe retinal degeneration (Hong et al., 2002). We generated two mutations in S5 that are proximal to the S4-S5 linker (S5px). One $\left(\operatorname{tr} p^{R 541 A}\right)$ caused a transient light response, which was distinct from other $\operatorname{trp}$ mutations in that it was dominant negative. However, the $\operatorname{trp} R 541 A /+$ phenotype was not as severe as the $\operatorname{tr} p^{343}$ null as the deactivation time during the light stimulus was much slower than in $\operatorname{trp} p^{343}$. We suggest that the TRP tetramers comprised of wild-type and $\mathrm{R} 541 \mathrm{~A}$ mutated subunits open in response to light, but TRP $\mathrm{R}^{\mathrm{R} 41 \mathrm{~A}}$ subunits cause the channels to close during light stimulation.

Residues corresponding to $\mathrm{TRP}^{\mathrm{R} 541}$ are conserved in all mammalian TRPCs except TRPC1. TRPC1 does not appear to be 
functional unless it is expressed with other TRPC subunits (Wang et al., 2020). Thus, the glutamine to arginine substitution in TRPC1 residue 548, which corresponds to R541 in fly TRP, might underlie the lack of TRPC1 activity.

Cerebellar ataxia results from the moonwalker mutation in mouse TRPC3, which changes a threonine to alanine (T635A) adjacent to the counterpart of arginine 541 in Drosophila TRP (Becker et al., 2009). Unlike $\mathrm{TRP}^{\mathrm{R} 541 \mathrm{~A}}$, which is dominant negative, TRPC $3^{\text {T635A }}$ is a dominant gain-of-function mutation. Nevertheless, the findings with $\mathrm{TRP}^{\mathrm{R} 541 \mathrm{~A}}$ and TRPC3 ${ }^{\mathrm{T} 635 \mathrm{~A}}$ highlight a critical role of $55 \mathrm{px}$ in channel regulation.

Surprisingly, the dominant-negative $\operatorname{trp} p^{R 41 A} /+$ phenotype was suppressed by trp ${ }^{S 538 A}$. We propose that S538A counters the effect of R541A because S538A slows channel inactivation whereas R541A greatly accelerates channel closing. The suppression of the mutant phenotype in $\operatorname{trp} p^{R 541 A} / \operatorname{trp} p^{S 538 A}$ occurs although the mutations in the S4-S5 linker and the S5px region are on different subunits. These results support the model that the S4-S5 linker of one subunit interacts with S5 of another (Hofmann et al., 2017). These results also support the concept that TRP channel gating depends on the concerted action of all subunits, consistent with a Monod-Wyman-Changeux model (Changeux and Christopoulos, 2016) as proposed for TRPM8 (Voets et al., 2007).

\section{TRP box 1 and gating of TRP}

The structures of TRP channels indicate that the $\sim 25$ amino acid $\alpha$-helical TRP domain lies perpendicular to the lipid bilayer and is in close contact with the S4-S5 linker (Liao et al., 2013; Romero-Romero et al., 2017; Fan et al., 2018). This domain includes a six amino acid TRP box 1 , which is invariant in all fly (675-EWKFAR) and human TRPCs, and TRP box 2, which includes multiple residues invariant in TRPCs (693-TIPPPFN in fly TRP; invariant residues are underlined).

TRPs with changes in TRP box 1 and in the linker between TRP boxes 1 and 2 were mostly expressed at high levels and localized normally (TRP ${ }^{\mathrm{MSN}}$ proteins), enabling us to assess the impact of the substitutions on TRP function. Four of five of these TRP ${ }^{M S N}$ proteins displayed transient ERGs indicating that the TRP box 1 and TRP box 1-2 linker are important for channel activity. Three $\operatorname{trp} p^{M S N}$ mutations caused less severe deactivation during the light response than the $\operatorname{trp}{ }^{343}$ null, suggesting that the alanine substitutions lower but do not eliminate the probability of opening. Structural modeling based on cryo-EM structures indicates an interaction between R541 and E675, underscoring the critical roles for
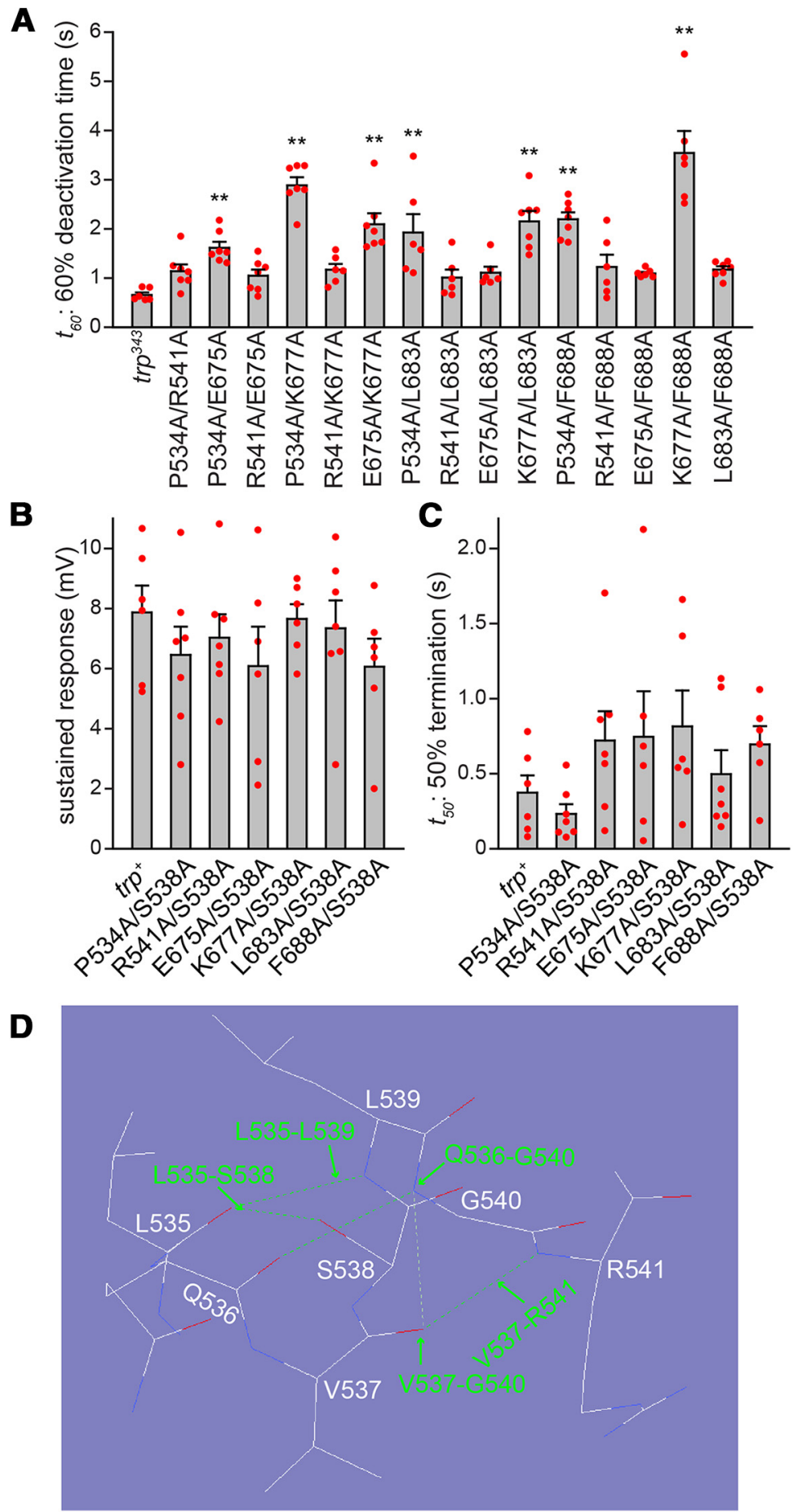

Figure 7. Quantification of ERGs of trans-heterozygous flies, shown in Figure 6. A, Quantification of the time required for $60 \%$ return to the baseline $\left(t_{60}\right)$ of the indicated trans-heterozygous combinations. Comparisons are to $\operatorname{trp}^{343}$. B, Amplitude of sustained depolarization responses at the end of the $10 \mathrm{~s}$ light stimulus. $C$, Time required for $50 \%$ return of the ERG response after cessation of the light stimulus $\left(t_{50}\right) . n \geq$ 6. Means \pm SEM. $D$, The backbones and side chains of L535 to R541 showing local hydrogen bonds between these residues, indicated by arrows.

these residues in mediating the interaction between the S4-S5 linker and TRP box 1 for TRP gating in photoreceptor cells.

The findings that mutations in the Drosophila TRP S4-S5 linker and TRP box 1 have an impact on the channel opening and closing in photoreceptor cells are intriguing in view of evidence that a lipid binds to a pocket in the related mammalian TRP channel (TRPC3), which is lined by the S4-S5 linker and 
TRP box 1 (Fan et al., 2018). Multiple studies implicate lipids such as diacylglycerol in the gating of TRPC channels including Drosophila TRP (Chyb et al., 1999; Hardie, 2003; Itsuki et al., 2012; Delgado et al., 2014; Curcic et al., 2019). Thus, interactions with the S4-S5 linker and TRP box 1 may underlie the effects of lipids on the activity of Drosophila TRP, as has been proposed previously for TRPC3 (Fan et al., 2018).

Surprisingly, the only mutation in TRP box 1 that had no impact on function replaced tryptophan 676 (W676A) in the EWKFAR motif. This was unexpected because W676 is invariant in all TRPC, TRPV, TRPN, and TRPM channels (Petrella and Karplus, 2004). Moreover, the structures of TRPs indicate interactions between this tryptophan and the S4-S5 linker or the Nterminal helix-loop-helix and that these interactions stabilize the closed conformation of some TRPs (Teng et al., 2015; Duan et al., 2018). Furthermore, mutation of the equivalent tryptophan in TRPV3 underlies some forms of Olmsted syndrome (Lin et al., 2012). However, structural modeling of Drosophila TRP indicates that W676 does not interface with these other domains. Rather, E675 appears to mediate an interaction between the TRP domain and the S4-S5 linker, whereas R680 of the TRP domains appears to interact with Q311 in the N-terminal helix-loop-helix.

\section{TRP box 2 and role in protein folding}

We suggest that TRP box 2 is required for protein folding. Substitutions of any of three invariant residues in TRP box 2 (P697A, F698A and N699A) cause low expression. These residues make up a radical turn, which may stabilize intersubunit interactions between coiled-coil domains necessary for subunit assembly (Duan et al., 2018). Disruption of subunit assembly would then lead to misfolding of single subunits that are subsequently degraded.

In conclusion, this work, which was built on the structures of TRP channels (Hofmann et al., 2017; Chen et al., 2020; Pumroy et al., 2020; Wang et al., 2020), revealed insights into TRP activation, intrasubunit interactions, and protein folding through a mutational analysis of the classical TRP in its native contextDrosophila photoreceptor cells. We suggest that these findings will form the basis for refining current models regarding TRP regulation and folding, and motivate future experiments on mammalian TRPs.

\section{References}

Beck A, Speicher T, Stoerger C, Sell T, Dettmer V, Jusoh SA, Abdulmughni A, Cavalié A, Philipp SE, Zhu MX, Helms V, Wissenbach U, Flockerzi V (2013) Conserved gating elements in TRPC4 and TRPC5 channels. J Biol Chem 288:19471-19483.

Becker EB, Oliver PL, Glitsch MD, Banks GT, Achilli F, Hardy A, Nolan PM, Fisher EM, Davies KE (2009) A point mutation in TRPC3 causes abnormal Purkinje cell development and cerebellar ataxia in moonwalker mice. Proc Natl Acad Sci U S A 106:6706-6711.

Bischof J, Maeda RK, Hediger M, Karch F, Basler K (2007) An optimized transgenesis system for Drosophila using germ-line-specific ФC31 integrases. Proc Natl Acad Sci U S A 104:3312-3317.

Changeux JP, Christopoulos A (2016) Allosteric modulation as a unifying mechanism for receptor function and regulation. Cell 166:1084-1102.

Chen X, Sooch G, Demaree IS, White FA, Obukhov AG (2020) Transient receptor potential canonical (TRPC) channels: then and now. Cells 9:1983.

Chen Z, Chen HC, Montell C (2015) TRP and rhodopsin transport depends on dual XPORT ER chaperones encoded by an operon. Cell Rep 13:573584.

Chevesich J, Kreuz AJ, Montell C (1997) Requirement for the PDZ domain protein, INAD, for localization of the TRP store-operated channel to a signaling complex. Neuron 18:95-105.
Chyb S, Raghu P, Hardie RC (1999) Polyunsaturated fatty acids activate the Drosophila light-sensitive channels TRP and TRPL. Nature 397:255-259.

Cosens DJ, Manning A (1969) Abnormal electroretinogram from a Drosophila mutant. Nature 224:285-287.

Curcic S, Schober R, Schindl R, Groschner K (2019) TRPC-mediated $\mathrm{Ca}^{2+}$ signaling and control of cellular functions. Semin Cell Dev Biol 94:28-39.

Delgado R, Muñoz Y, Peña-Cortés H, Giavalisco P, Bacigalupo J (2014) Diacylglycerol activates the light-dependent channel TRP in the photosensitive microvilli of Drosophila melanogaster photoreceptors. J Neurosci 34:6679-6686.

Duan J, Li J, Zeng B, Chen GL, Peng X, Zhang Y, Wang J, Clapham DE, Li Z, Zhang J (2018) Structure of the mouse TRPC4 ion channel. Nat Commun 9:3102.

Fan C, Choi W, Sun W, Du J, Lü W (2018) Structure of the human lipidgated cation channel TRPC3. eLife 7:e36852.

Gees M, Owsianik G, Nilius B, Voets T (2012) TRP channels. Compr Physiol 2:563-608.

Hardie RC (2003) Regulation of TRP channels via lipid second messengers. Annu Rev Physiol 65:735-759.

Hardie RC, Minke B (1992) The trp gene is essential for a light-activated $\mathrm{Ca}^{2+}$ channel in Drosophila photoreceptors. Neuron 8:643-651.

Hardie RC, Raghu P, Moore S, Juusola M, Baines A, Sweeney ST (2001) Calcium influx via TRP channels is required to maintain $\mathrm{PIP}_{2}$ levels in Drosophila photoreceptors. Neuron 30:149-159.

Hilton JK, Kim M, Van Horn WD (2019) Structural and evolutionary insights point to allosteric regulation of TRP ion channels. Acc Chem Res 52:1643-1652.

Hofmann L, Wang H, Zheng W, Philipp SE, Hidalgo P, Cavalie A, Chen XZ, Beck A, Flockerzi V (2017) The S4-S5 linker-gearbox of TRP channel gating. Cell Calcium 67:156-165.

Hong YS, Park S, Geng C, Baek K, Bowman JD, Yoon J, Pak WL (2002) Single amino acid change in the fifth transmembrane segment of the TRP $\mathrm{Ca}^{2+}$ channel causes massive degeneration of photoreceptors. J Biol Chem 277:33884-33889.

Itsuki K, Imai Y, Okamura Y, Abe K, Inoue R, Mori MX (2012) Voltage-sensing phosphatase reveals temporal regulation of TRPC3/C6/C7 channels by membrane phosphoinositides. Channels (Austin) 6:206-209.

Kremeyer B, Lopera F, Cox JJ, Momin A, Rugiero F, Marsh S, Woods CG, Jones NG, Paterson KJ, Fricker FR, Villegas A, Acosta N, Pineda-Trujillo NG, Ramírez JD, Zea J, Burley MW, Bedoya G, Bennett DL, Wood JN, Ruiz-Linares A (2010) A gain-of-function mutation in TRPA1 causes familial episodic pain syndrome. Neuron 66:671-680.

Lai-Cheong JE, Sethuraman G, Ramam M, Stone K, Simpson MA, McGrath JA (2012) Recurrent heterozygous missense mutation, p.Gly573Ser, in the TRPV3 gene in an Indian boy with sporadic Olmsted syndrome. Br J Dermatol 167:440-442.

Liao M, Cao E, Julius D, Cheng Y (2013) Structure of the TRPV1 ion channel determined by electron cryo-microscopy. Nature 504:107-112.

Lin Z, Chen Q, Lee M, Cao X, Zhang J, Ma D, Chen L, Hu X, Wang H, Wang $X$, Zhang P, Liu X, Guan L, Tang Y, Yang H, Tu P, Bu D, Zhu X, Wang KW, Li R, Yang Y (2012) Exome sequencing reveals mutations in TRPV3 as a cause of Olmsted syndrome. Am J Hum Genet 90:558-564.

Montell C (2001) Physiology, phylogeny and functions of the TRP superfamily of cation channels. Sci STKE 2001:re1.

Montell C (2005) The TRP superfamily of cation channels. Sci STKE 2005:re3.

Montell C (2012) Drosophila visual transduction. Trends Neurosci 35:356-363.

Montell C, Rubin GM (1989) Molecular characterization of the Drosophila trp locus: a putative integral membrane protein required for phototransduction. Neuron 2:1313-1323.

Montell C, Jones K, Hafen E, Rubin G (1985) Rescue of the Drosophila phototransduction mutation trp by germline transformation. Science 230:1040-1043.

Moore C, Gupta R, Jordt SE, Chen Y, Liedtke WB (2018) Regulation of pain and itch by TRP channels. Neurosci Bull 34:120-142.

Ni C, Yan M, Zhang J, Cheng R, Liang J, Deng D, Wang Z, Li M, Yao Z (2016) A novel mutation in TRPV3 gene causes atypical familial Olmsted syndrome. Sci Rep 6:21815.

Petrella RJ, Karplus M (2004) The role of carbon-donor hydrogen bonds in stabilizing tryptophan conformations. Proteins 54:716-726.

Pumroy RA, Fluck EC, 3rd, Ahmed T, Moiseenkova-Bell VY (2020) Structural insights into the gating mechanisms of TRPV channels. Cell Calcium 87:102168. 
Romero-Romero S, Gomez Lagunas F, Balleza D (2017) Side chain flexibility and coupling between the S4-S5 linker and the TRP domain in thermo-sensitive TRP channels: insights from protein modeling. Proteins 85:630-646.

Stallmeyer B, Zumhagen S, Denjoy I, Duthoit G, Hebert JL, Ferrer X, Maugenre S, Schmitz W, Kirchhefer U, Schulze-Bahr E, Guicheney P, Schulze-Bahr E (2012) Mutational spectrum in the $\mathrm{Ca}^{2+}$-activated cation channel gene TRPM4 in patients with cardiac conductance disturbances. Hum Mutat 33:109-117.

Teng J, Loukin SH, Anishkin A, Kung C (2015) L596-W733 bond between the start of the S4-S5 linker and the TRP box stabilizes the closed state of TRPV4 channel. Proc Natl Acad Sci U S A 112:3386-3391.

Teng J, Loukin SH, Anishkin A, Kung C (2016) A competing hydrophobic tug on L596 to the membrane core unlatches S4-S5 linker elbow from TRP helix and allows TRPV4 channel to open. Proc Natl Acad Sci U S A 113:11847-11852.

Vangeel L, Voets T (2019) Transient receptor potential channels and calcium signaling. Cold Spring Harb Perspect Biol 11:a035048.

Venkatachalam K, Montell C (2007) TRP channels. Annu Rev Biochem 76:387-417.

Voets T, Owsianik G, Janssens A, Talavera K, Nilius B (2007) TRPM8 voltage sensor mutants reveal a mechanism for integrating thermal and chemical stimuli. Nat Chem Biol 3:174-182.

Wang H, Cheng X, Tian J, Xiao Y, Tian T, Xu F, Hong X, Zhu MX (2020) TRPC channels: structure, function, regulation and recent advances in small molecular probes. Pharmacol Ther 209:107497.

Wang T, Jiao Y, Montell C (2005) Dissecting independent channel and scaffolding roles of the Drosophila transient receptor potential channel. J Cell Biol 171:685-694.
Waterhouse A, Bertoni M, Bienert S, Studer G, Tauriello G, Gumienny R, Heer FT, de Beer TAP, Rempfer C, Bordoli L, Lepore R, Schwede T (2018) SWISS-MODEL: homology modelling of protein structures and complexes. Nucleic Acids Res 46:W296-W303.

Wes PD, Chevesich J, Jeromin A, Rosenberg C, Stetten G, Montell C (1995) TRPC1, a human homolog of a Drosophila store-operated channel. Proc Natl Acad Sci U S A 92:9652-9656.

Wes PD, Xu X-ZS, Li H-S, Chien F, Doberstein SK, Montell C (1999) Termination of phototransduction requires binding of the NINAC myosin III and the PDZ protein INAD. Nat Neurosci 2:447-453.

Xian W, Wang H, Moretti A, Laugwitz KL, Flockerzi V, Lipp P (2020) Domain zipping and unzipping modulates TRPM4's properties in human cardiac conduction disease. FASEB J 34:12114-12126.

Xiao R, Xu XZ (2009) Function and regulation of TRP family channels in C. elegans. Pflugers Arch 458:851-860.

Xiao R, Tian J, Tang J, Zhu MX (2008) The TRPV3 mutation associated with the hairless phenotype in rodents is constitutively active. Cell Calcium 43:334-343.

Zhong W, Hu L, Cao X, Zhao J, Zhang X, Lee M, Wang H, Zhang J, Chen Q, Feng C, Duo L, Wang X, Tang L, Lin Z, Yang Y (2021) Genotype-phenotype correlation of TRPV3-related Olmsted syndrome. J Invest Dermatol 141:545-554.

Zhu X, Chu PB, Peyton M, Birnbaumer L (1995) Molecular cloning of a widely expressed human homologue for the Drosophila trp gene. FEBS Lett 373:193-198.

Zíma V, Witschas K, Hynkova A, Zímová L, Barvík I, Vlachova V (2015) Structural modeling and patch-clamp analysis of pain-related mutation TRPA1-N855S reveal inter-subunit salt bridges stabilizing the channel open state. Neuropharmacology 93:294-307. 